\title{
EXPOSURE TO SWINE HOUSING DUST MODULATES MACROPHAGE MORPHOLOGY AND FUNCTION
}

\author{
Ruth J. Pender, Radiah C. Minor, Steven L. Hurley, \\ Dawn R. Conklin and Jenora T. Waterman
}

Department of Animal Sciences, North Carolina Agricultural and Technical State University, Greensboro, USA

Received 2013-09-17; Revised 2014-03-08; Accepted 2014-03-25

\begin{abstract}
Swine Confinement Facility (SCF) dust consists of a complex mixture of feed grain particles, bacterial components, organic particulates and gases. When these particles are inhaled they deposit along the respiratory tract and mediate respiratory symptoms and disease in swine farmers and facility workers. Macrophages ingest and eliminate microbes and debris under chronic conditions; however, the role of macrophages in agricultural-related respiratory disease has not been fully elucidated. The goal was to evaluate the hypothesis that chronic exposure to SCF dust causes inflammation by modulating pulmonary protein levels and macrophage function. Balb/c mice were exposed to 5, 12.5 and 25\% SCF Dust Extract (DE) via nebulization $30 \mathrm{~min} /$ day five days a week, for eight weeks with weekends excluded. Bronchoalveolar Lavage Fluid (BALF) was collected and analyzed for protein concentration, leukocyte distribution and macrophage morphology. For comparison, THP-1 monocytic cells were exposed to 0.1$10 \%$ DE overnight and evaluated for phagocytosis and reactive oxygen species production. Repeated exposure to DE via nebulizer caused a significant increase in protein concentration and inflammatory cell number, namely macrophages, in a dose-dependent manner within the lung as compared to controls. Macrophages with pseudopods and vacuoles were the most abundant leukocytes within BALF of mice exposed to DE. Similarly, in vitro studies with $10 \%$ DE treated THP-1 cells revealed enhanced phagocytosis $(\mathrm{p}<0.05)$, pseudopodia and vacuolization following exposure to compared to control cells. In addition, there were time- and dose-dependent increases of intracellular ROS production by THP-1 cells exposed to 5 and $10 \%$ DE compared to control $(\mathrm{p}<0.01)$. These findings indicate repeated, long-term inhalation of swine confinement facility dust may mediate chronic airway and lung inflammation through modulation of protein concentration and macrophage function. The aerosolized dust-mouse inhalation model presented here may offer a good tool for studying particle mediated chronic inflammation of the tracheobronchial tree and lungs.
\end{abstract}

Keywords: Nebulizer, Swine Housing Dust, Macrophage, Phagocytosis, Reactive Oxygen Species

\section{INTRODUCTION}

In an effort to meet growing consumer demand, animal production has shifted from pasture-based approached to large Concentrated Animal Feeding Operations (CAFOs). A myriad of respiratory complications arise from acute or chronic inhalation of organic dusts from CAFOs including non-allergic asthma, rhinitis, sinusitis, chronic bronchitis, Chronic Obstructive Pulmonary Disease (COPD) and organic dust toxic syndrome as reviewed by May et al. (2012). Swine facility workers may experience occupationalhazards from inhalation of high levels of organic dusts which consist of feed particles, fecal material, endotoxins, ammonia odors, organic dust, gases and other dried windborne material from animals, microbes and fungi (Donham et al., 2006; Rylander, 2010). Inhalation of endotoxins and carbon dioxide at levels above the recommended health threshold limits (McDonnell et al., 2008) can lead to acute and chronic

Corresponding Author: Jenora T. Waterman, Department of Animal Sciences, North Carolina Agricultural and Technical State University, 1601 East Market Street, Greensboro NC 27411, USA Tel: (336) 285-4815 Fax: (336) 334-7288 
airway inflammation can occur (Schierl et al., 2007) which may lead to loss of lung function, as measured by the decrease in forced expiratory air in one second or less (Costa et al., 2007). After frequent exposure to Swine Confinement Facility (SCF) dust, physiological effects can develop including pulmonary inflammation, severe asthma, chronic respiratory diseases and bronchoconstriction (Demanche et al., 2009). Inhalation of endotoxins and carbon dioxide at levels above the recommended health threshold limits (McDonnell et al., 2008) chronic airway inflammation can occur (Schierl et al., 2007) which may lead to loss of lung function, as measured by the decrease in forced expiratory air in one second or less (Costa et al., 2007). After frequent exposure to Swine Confinement Facility (SCF) dust, physiological effects can develop, including pulmonary inflammation, severe asthma, chronic respiratory diseases and bronchoconstriction (Demanche et al., 2009).

Macrophages are mature myeloid cells that originate from differentiated monocytes and circulate through vasculature or reside within tissues. Although lung tissue macrophages reside in gas-exchange regions of the lung and modulate inflammation therein; tissue inflammatory responses in general signal recruitment of more macrophages to the site of injury (Rodero and Khosrotehrani, 2010). Macrophages and other specialized cells remove pathogens, dead cell remnants and other debris via phagocytosis, a well-orchestrated process involving encapsulation and internalization of large particles (Underhill and Goodridge, 2012). However, the role of macrophages in chronic lung disease as seen in agricultural occupational settings is not fully understood.

A great deal of attention has been devoted to elucidating acute inflammation responses that follow single or short-term exposure to CAFO-style organic dust; however, fewer models describe the effects of chronic or long-term exposures which may require extended exposure durations (e.g., several hours) and frequency (e.g., 8 weeks or several months). The purpose of the present study was to characterize the effect of long-term or chronic organic dust exposure on airway inflammation in mice that were exposed to SCF Dust Extract (DE), 30 min a day, five days per week for eight weeks via nebulizer. After the exposure was completed, mice were euthanized and bronchoalveolar washing was performed to evaluate protein concentrations, leukocyte numbers and cell morphology to characterize inflammation of the lungs. Cell culture comparisons with THP-1 cells were conducted to evaluate DE mediated modulation of macrophage function in vitro. We report here that long-term repeated inhalation of aerosolized SCF dust enhances bronchoalveolar fluid protein concentration and formation of pseudopodia and vacuolation by airway macrophages in vivo. Correspondingly, increases in pseudopodia and vacuolation, enhanced phagocytosis and elevated ROS production was observed from THP-1 following DE exposure in vitro.

\section{MATERIALS AND METHODS}

\subsection{Reagents}

Penicillin-Streptomycin solution, 2-Mercapto-Ethanol And Dimethyl Sulfoxide (DMSO) were obtained from Sigma-Aldrich (St. Louis, MO, USA). Heat-inactivated Fetal Bovine Serum (FBS) was obtained from Atlanta Biologicals (Atlanta, GA, USA). Lipopolysaccharide (LPS) from Escherichia coli 0111: B4, RPMI 1640 and HEPES were purchased from Thermo Fisher Scientific (Waltham, MA, USA). Phorbol 12-Myristate 13-Acetate (PMA) was obtained from Tocris (Ellisville, MO, USA). 5-(and-6)-chloromethyl '7'-dichloro dyhydrogluoresceindiacetate, acetyl ester (DCF-DA) was purchased from Invitrogen Molecular Probes (Eugene, OR, USA). Black well, clear bottom tissue culture plates were from Corning Life Sciences (Tewksbury, MA, USA). The THP-1 cell line was obtained from the American Type Cell Collection (Rockville, MD, USA).

\subsection{Dust collection and Extract Preparation for in Vivo and in Vitro Studies}

The dust extract was prepared using a protocol similar to Poole et al. (2009b). Briefly, several grams of settled dust from raised surfaces were collected from the gestation and farrowing rooms at North Carolina $\mathrm{A}$ and $\mathrm{T}$ State University's Swine Research Unit and brushed into a zip top bag. A 1:10 (w/v) ratio of dust and Phosphate Buffer Saline (PBS) without calcium and magnesium or Hank's Balanced Salt Solution (HBSS) was prepared. The mixture was vortexed for approximately $1 \mathrm{~m}$ and incubated at room temperature for $1 \mathrm{~h}$. The mixture was centrifuged in an Eppendorf 5810 R (Hauppauge, NY) centrifuge for $10 \mathrm{~min}$, at $948 \times \mathrm{g}$ at room temperature and the supernatant was transferred to a new tube. The mixture was centrifuged again to remove any leftover dust particles from the first centrifugtion. The mixture was sterilized by filtration $(0.22$ $\mu \mathrm{m})$ and the dust extract was used immediately.

\subsection{Mice and Nebulizer Dust Extract Exposure}

Female Balb/c mice of ages 6 to 7 weeks (Harlan Laboratories, Indianapolis, IN) were maintained at the North Carolina A and T State University (NCAT) Laboratory Animal Resource Unit under standard housing conditions. Mice received water and standard 
rodent chow ad libitum for the entire course of the study and were weighed weekly. Mice were randomly placed into 1 of 4 treatment groups ( $n=6$ per groups): Group 1 , aerosolization exposure to PBS; and Groups 2, 3 and 4, aerosolization exposure to dust extract (5, 12.5 and $25 \%$ ), respectively. Our nebulizer SCF dust exposure model is a modified version of a grain dust exposure model (George et al., 2001). In the present study, mice were placed into an exposure chamber connected to a treatment solution-filled nebulizer, which itself was connected to an air pump. To simulated, repeat occupational exposure to swine dust, mice were exposed to DE or PBS for 30 min per day, 5 days a week for 8 weeks with weekends excluded according to their respective treatment groups described above. This procedure was performed twice with three mice per group in each trial for a total of six mice per group. All experimental protocols were reviewed and approved by the North Carolina A and $\mathrm{T}$ State University Institutional Animal Care and Use Committee and all protocols conformed to the Guide for the Care and Use of Laboratory Animals (NRC, 2011).

\subsection{Bronchoalveolar Lavage Collection and Differential Cell Counting}

After the 8 week treatment period, mice were euthanized by xylazine injection $\left(100 \mathrm{mg} \mathrm{mL}^{-1}\right)$ and death was assured by auscultation of the lungs. Bronchoalveolar Lavage (BAL) was collected via the trachea by washing lungs a total of three times with 1 ml-of ice cold PBS; a method similar to Minor et al. (2012). Supernatant collected from the first lavage was used for protein assays. Cells from all three lavage washings were pooled. Cell concentration and viability was determined for BAL fluid from pooled samples collected from each mouse by trypan blue dye exclusion. Briefly, in a microcentrifuge tube, 10 $\mu \mathrm{L}$ of trypan blue was added to $10 \mu \mathrm{L}$ of cells and the cells were allowed to sit for two minutes. Cells $(\sim 2,000$ in $100 \mu \mathrm{L}$ ) were collected on slides via cytocentrifugation at $55 \times \mathrm{g}$ for $5 \mathrm{~min}$ using a CytoSpin 4 (Thermo Fisher Scientific). Hema 3 System (Fisher Scientific Company, Suwanee, GA) was used to stain cells according to manufacturer's protocol. The stained leukocytes were counted and differentiated based on color and morphology using a Leica Model DME Microscope with an oil emersion lens (Leica Microsystems, Shanghai, China) counting a total of 100 cells.

\subsection{Cell Culture and Differentiation}

The promonocytic THP-1 cell line (ATCC) was maintained at a density of $2-8 \times 10^{5}$ cells $/ \mathrm{mL}$ in THP- 1 complete media which consisted of RPMI-1640 supplemented with $1 \%$ Penicillin $(10,000 \quad \mathrm{U})$ Streptomycin $(10 \mathrm{mg}), 0.5 \mathrm{mg} \mathrm{mL}^{-1}$ gentamicin, $200 \mathrm{mM}$ L-glutamine, $10 \mathrm{mM}$ HEPES, $50 \mathrm{mM}$ 2-mercaptoethanol and $10 \%$ heat inactivated Fetal Calf Serum (FCS), at $37^{\circ} \mathrm{C}$ in humidified air mixed with $5 \% \mathrm{CO}_{2}$. For differentiation, THP-1 cells were stimulated with $160 \mathrm{nM}$ PMA for 24 or $48 \mathrm{~h}$. Non-adherent cells were removed; cell surfaces were washed with PBS and fresh media with or without treatment components was added as described below. For THP-1 cell imaging, cells were seeded onto Millicell EZ glass slides (EMD Millipore, Billerica, MA) at a density of $10^{4}$ cells $/ \mathrm{cm}^{2}$ and exposed to DE as described. To end the experiment media was removed and adherent cells were washed with PBS, slide chambers were aspirated, chambers were then detached and slides were stained with Hema 3 Diff Quick (Thermo Fisher Scientific) according to manufacturer's instructions. Cells were visualized using a Zeiss Axio Imager $\mathrm{m} 2 \mathrm{~m}$ Optical Microscope (Carl Zeiss Microscopy $\mathrm{GmbH}$, Jena, Germany) at $1000 \mathrm{X}$ magnification.

\subsection{Measurement of Phagocytosis}

Phagocytosis was assessed using the phagocytosis assay kit (FITC) (Cayman Chemical, Ann Arbor, MI). Briefly, THP-1 cells, plated in triplicate at a density of $2 \times 10^{5}$ cells $\mathrm{mL}^{-1}$ in the absence or presence of PMA [160 $\mathrm{nM}]$, LPS $\left[1 \mu \mathrm{g} \mathrm{mL}^{-1}\right]$, or DE $[0.1,1.0$ and $10 \%]$ with and without latex beads coated with FITC-labeled rabbit IgG. After $24 \mathrm{~h}$ in culture at $37^{\circ} \mathrm{C}$, the uptake of the beads into cells was detected using an Accuri C6 flow cytometer (BD Biosciences, Franklin Lakes, NJ.). Data were analyzed using FCS express (DeNovo Software, Los Angeles, CA).

\subsection{Measurement of Reactive Oxygen Species Production}

Intracellular hydrogen peroxide generation was measured using DCF-DA (Molecular Probes). Briefly, $7.5 \times 0^{4}$ THP-1 cells $/ \mathrm{mL}$ were plated in black well-clear bottom 96-well plates (Corning Life Sciences, Corning, $\mathrm{NY}$ ) overnight in the absence or PMA [160 nM], LPS [1 $1 \mu \mathrm{g}$ $\left.\mathrm{mL}^{-1}\right]$, or DE $[0.1,1.0,5$ and $10 \%]$. Cells were loaded with DCF-DA $[10 \mu \mathrm{M}]$ for $1 \mathrm{~h}$ at $37^{\circ} \mathrm{C}$ and fluorescence was measured using a SpectroMax M5 microplate reader (Molecular Devices, Sunnyvale, CA) every 20 min for $2 \mathrm{~h}$ at $485 \mathrm{~nm}$ excitation and $538 \mathrm{~nm}$ emission. Fluorescence intensity results were expressed as arbitrary units.

\subsection{Statistical Analysis}

Two to three biological replicates, with at least three to six technical replicates were performed for each 
experiment. Data were analyzed for significance by oneway or repeated measures two-way Analysis Of Variance (ANOVA) with a threshold set at a p-value of 0.05 or below using Prism version 5.0 (GraphPad, La Jolla, CA) followed by Bonferroni post-test corrections, or as described. Data are shown as mean \pm Standard Error Measure (SEM), mean \pm Standard Deviation (SD) or as described.

\section{RESULTS}

\subsection{Inhalation of aerosolized DE Increases Protein Concentration and the Number of Macrophages with Pseudopods and Vacuoles in Mouse BALF}

Previous studies have shown intranasal instillation of DE [1-25\%] once daily for 2 weeks induces lung inflammation (Poole et al., 2009a). To examine the effect of long-term DE exposure, a mouse model was developed using a nebulizer to aerosolize PBS [0\%] or $\mathrm{DE}[5,12.5$ or $25 \%]$ for exposures. Mice inhaled aerosolized PBS or DE for $30 \mathrm{~min}$ a day for eight weeks with weekends excluded. At the end of the 8 week period, airways and lungs were washed to collect BALF. A Bradford assay was performed to determine the concentration of protein in BALF. There was a significant increase in BALF protein concentration in $12.5 \% \mathrm{DE}$ exposed mice compared to PBS-control (Fig. 1A).

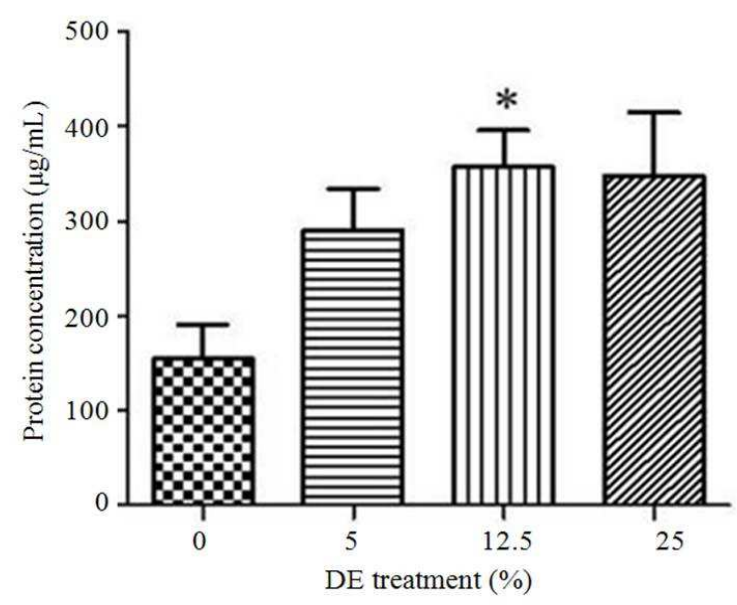

(A)
There was a trend for higher cell numbers in DEexposed mice (Fig. 1B); however, no significant differences were observed when treatment groups were compared to control by one-way analysis of variance $(\mathrm{p}>0.05)$. Figure 2 A-C shows leukocytes (lymphocytes, monocytes, neutrophils) found within BALF extracted from the lungs after exposure to various DE. The levels of these cells were low and there were no significant changes in the levels of these cells found. In contrast abundant macrophages within the BALF were detected (Fig. 3A). Although there was no difference in the percentage of macrophages between the treatment groups we identified macrophages with different morphologies; macrophages with pseudopods, macrophages with large vacuoles. Figure 3B depicts a representative image of macrophages from PBS-control mice. We observed higher levels of macrophages with pseudopods and vacuoles in DE-exposed mice as compared to control. There was a significant increase in macrophages with pseudopods $(\mathrm{p}<0.001)$ in mice that inhaled aerosolized DE compared to PBS-control (Fig. 4A).

Figure 4B is a representative phase contrast image of observed macrophages with pseudopods. There was also a dose-dependent increase in macrophages withvacuoles $(\mathrm{p}<0.01)$ in BALF of mice exposed to 12.5 and $25 \%$ DE compared to control mice (Fig. 5A). A representative image of vacuolated macrophages observed in DE exposed mice is shown in Fig. 5B.

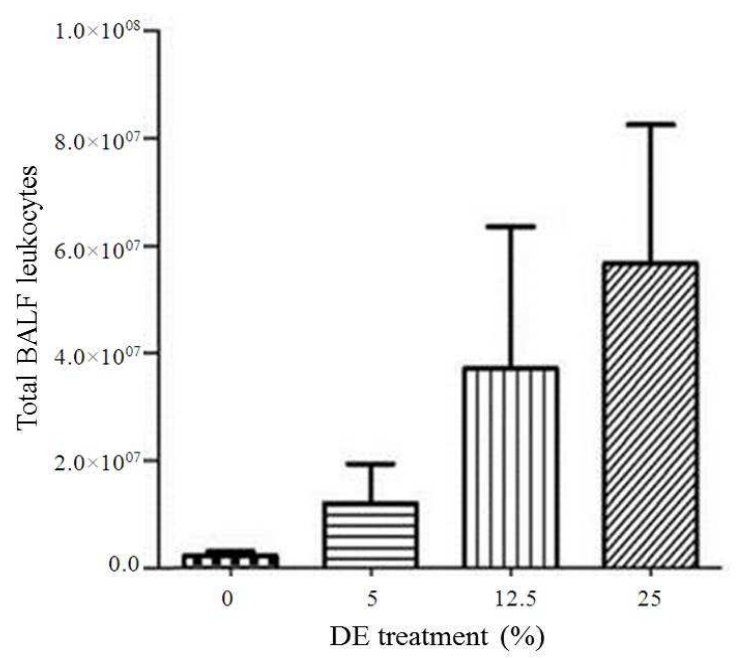

(B)

Fig. 1. Bronchoalveolar Lavage Fluid (BALF) protein concentration (A) and total leukocyte number (B) in lungs of mice delivered PBS or DE via nebulizer. Mice were exposed to PBS [0], 5, 12.5 or $25 \%$ DE ( $n=6$ each) via nebulization for 8 weeks. After the 8-week exposure period had ended, BALF was collected by washing lungs three times, cells were affixed to slides and differential cell counts were performed. Protein concentration was determined by Bradford assay. *p<0.05 for $12.5 \% \mathrm{DE}$ exposed vs. control (0\%) mice as compared by one-way analysis of variance paired with Bonferroni posttest corrections. Protein concentration data are expressed as mean \pm SEM and cell data are shown as mean \pm SD. $N=6$ each 


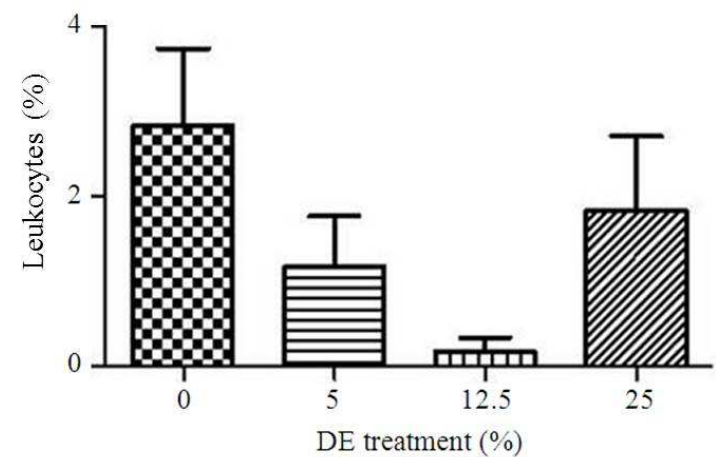

(A)

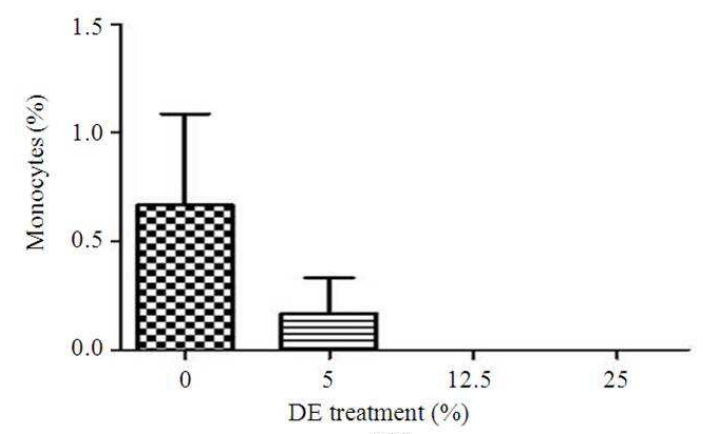

(B)

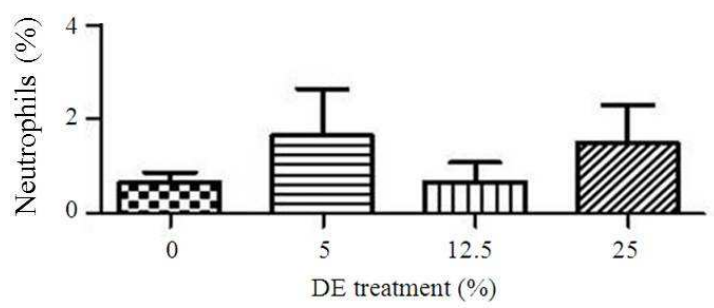

(C)

Fig. 2. Differential BALF leukocyte count. Mice were exposed to aerosolized PBS [0\%] or DE [5, 12.5 and $25 \%$ ] for 8 weeks. After 8-weeks, lungs were washed three times to collect BALF, cells were affixed to slides and differential cell counts were performed. (A) Lymphocytes; (B) Monocytes; and (C) Neutrophils were the predominate types of leukocytes present in BALF of mice. There were 6 mice per group. Data are shown as mean \pm SD

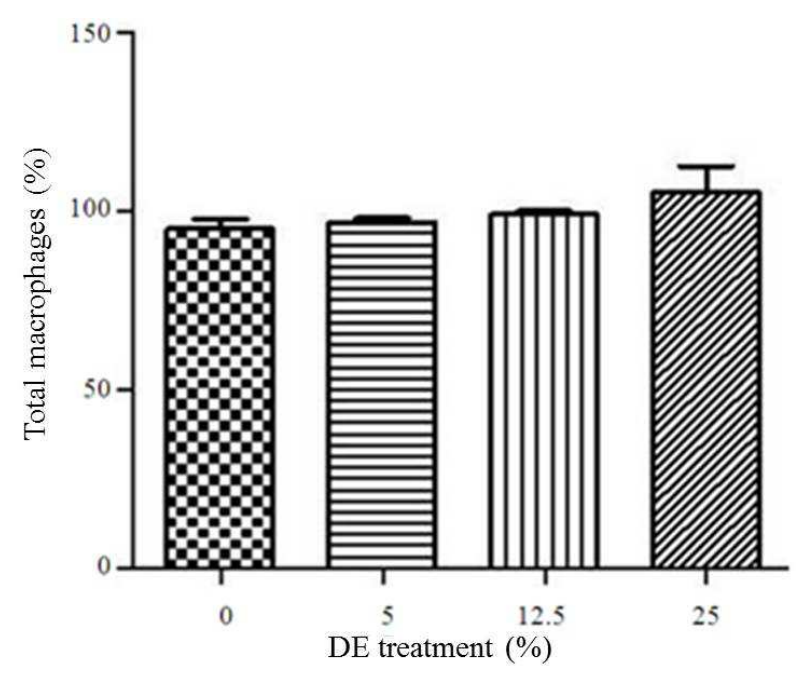

(A)

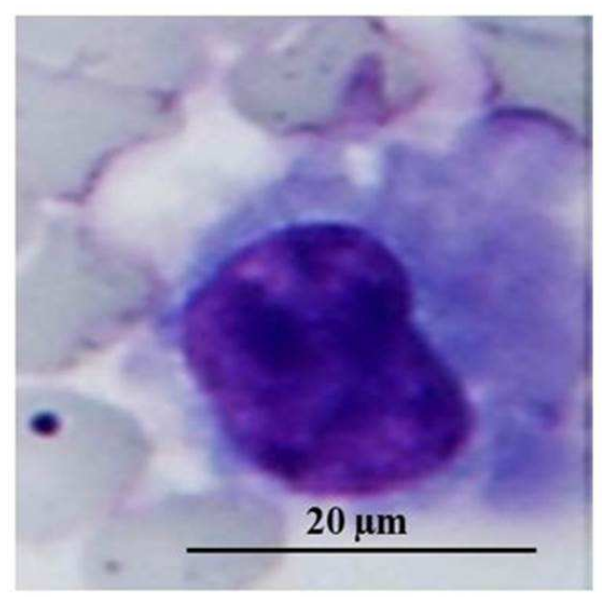

(B)

Fig. 3. Total macrophages present in BALF of DE-exposed mice. Mice were exposed to PBS [0\%] or DE [5, 12.5 and $25 \%]$ via inhalation for 8 weeks. After the 8-week period, lungs were washed three times to collect BALF; cells were attached to slides, stained and differentially counted for macrophages. (A) Total macrophages in BALF. (B) Representative micrograph of macrophages from BALF of mice. There were 6 animals per group. Data are presented as mean \pm SD. Magnification, 1000X; Scale bar $=20 \mu \mathrm{m}$ 


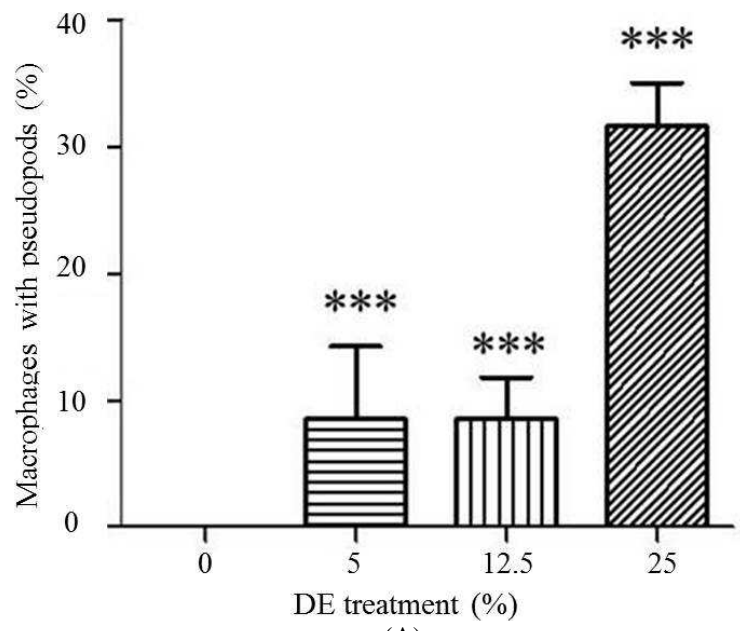

(A)

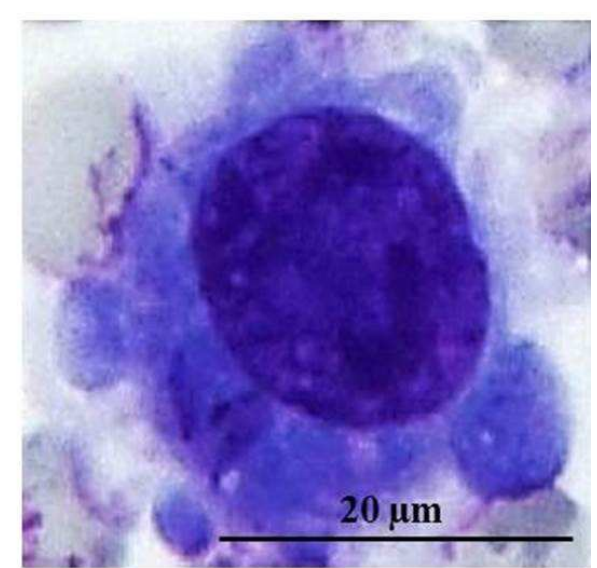

(B)

Fig. 4. Macrophages with pseudopodia present in BALF of DE-exposed Balb/c mice. A nebulizer was used to deliver aerosolized PBS [0\%] or DE [5, 12.5, 25\%] to mice ( $\mathrm{n}=6$ per group) for 2 months. After the 2 month period, BALF was obtained by washing lungs three times; cells were affixed to slides, stained and macrophages with pseudopods were counted. (A) Macrophages with pseudopods in BALF. (B) Representative micrograph of macrophages with pseudopodia in BALF of mice. $* * *, p<0.001$ for DE exposed vs. control $(0 \%)$ mice when compared by one-way analysis of variance paired with Bonferroni posttest corrections. Data are expressed as mean \pm SD. Magnification, 1000X; Scale bar $=20 \mu \mathrm{m}$

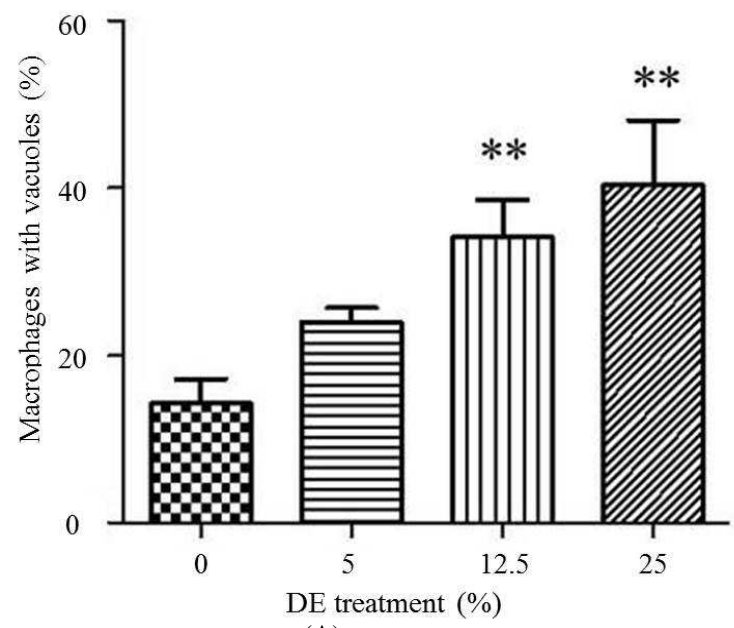

(A)

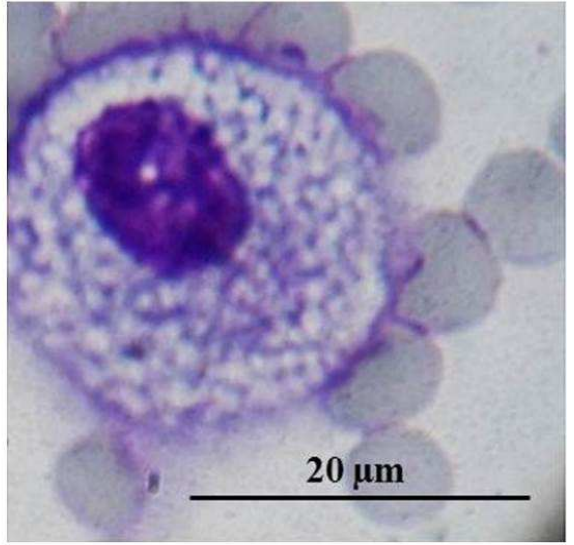

(B)

Fig. 5. Dust extract mediated-induction of macrophages with vacuoles in mouse BALF. Aerosolized PBS [0\%] or DE [5, 12.5, 25\%] was delivered to mice ( $n=6$ per group) for 8 weeks. After the end of 8 weeks, BALF was obtained from three lung washes; BALF cells were affixed to slides, stained and macrophages with vacuoles were counted. (A) Macrophages with vacuoles in BALF. (B) Representative phase contrast image of macrophages with vacuoles present in BALF of mice. **, $\mathrm{p}<0.01$ for 12.5 and $25 \%$ DE exposed vs.control $(0 \%)$ mice when compared by one-way analysis of variance paired with Bonferroni posttest corrections. Data are expressed as mean \pm SD. Magnification, 1000X; Scale bar $=20 \mu \mathrm{m}$

\subsection{Organic DE Enhances Phagocytosis and ROS Production by THP-1 cells}

To evaluate effects of organic DE on macrophage function, THP-1 cells were incubated with or without
PMA, LPS and escalating concentrations of DE for 24$48 \mathrm{~h}$. Visual inspection of THP-1 cells exposed to culture media or DE [0.1, 1 and $10 \%$ ] for $48 \mathrm{~h}$ revealed a morphological trend similar to observations of in vivo alveolar macrophages (Fig. 4 and 5) in that there 
appeared to be enhanced pseudopod and vacuole formation in DE-exposed cultures (Fig. 6). As shown in Fig. 6, panels A-D depict representative images of THP1 cells cultured in the presence of culture media only (Fig. 6A), or media contain $0.1 \%$ DE (Fig. 6B), $1 \%$ DE (Fig. 6C) or 10\% DE (Fig. 6D).

Exposure to occupations dust has been shown to modulate macrophage function and phagocytosis (Meo et al., 2008; Poole et al., 2009a), therefore we assessed phagocytosis of THP-1 cells cultured in the presence of various concentrations of DE. Fig. 7 shows enhanced phagocytosis by THP-1 cells following exposure to dust suggesting that dust extracts promoted phagocytosis in a dose-dependent manner (Fig. 7A and B). It is well accepted that phagocytes generate large amounts of superoxide and hydrogen peroxide when stimulated (Robinson, 2008) and here we demonstrate time and concentration dependent increases in intracellular hydrogen peroxide production by THP-1 cells following exposure to SCF DE (Fig. 8).
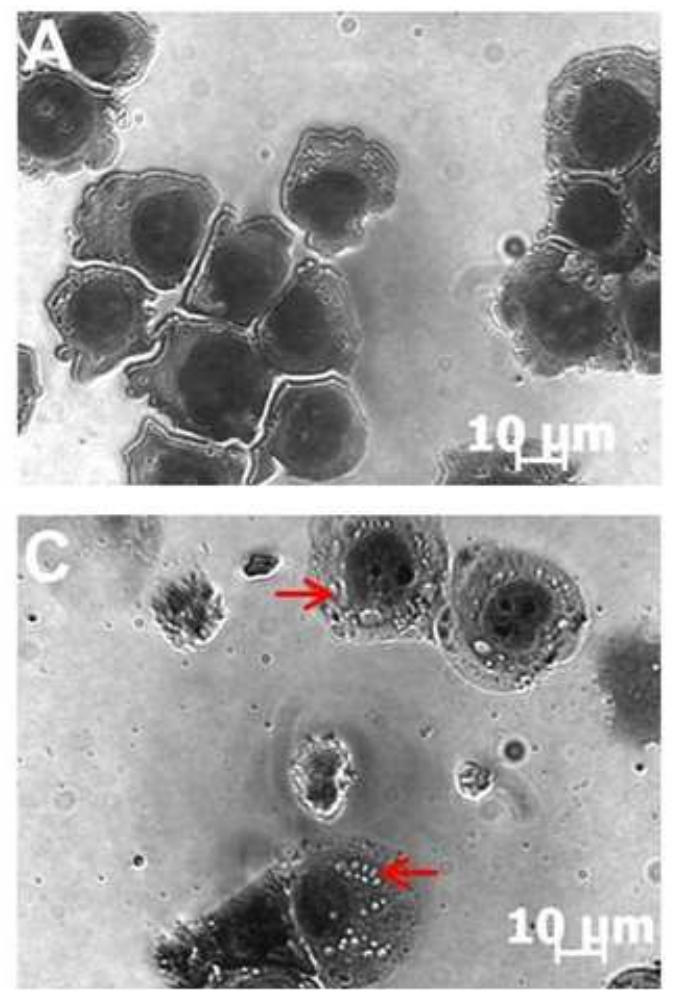

\section{DISCUSSION}

Swine confinement facilities and Concentrated Animal Feeding Operations (CAFOs) harbor high levels of dust consisting largely of feed particles, fecal matter, animal dander microbes and their products including lipopolysaccharide and peptidoglycan (May et al., 2012). CAFO dust containing LPS can cause severe inflammatory response (Hollingsworth et al., 2007). Acute inflammation was observed in Spague-Dawley rats exposed to swine barn dust or ambient air for $8 \mathrm{~h}$ as determined by enhanced levels of pulmonary intravascular monocytes/macrophages found in bronchoalveolar lavage fluid (Gamage et al., 2007). Acute lung inflammation obesrved in animal models of single exposure to elements of swine barn air is consistent with effects reported from similar human single exposure studies (Charavaryamath and Singh, 2006). However, farmers and animals of SCF are continually exposed to this environment.
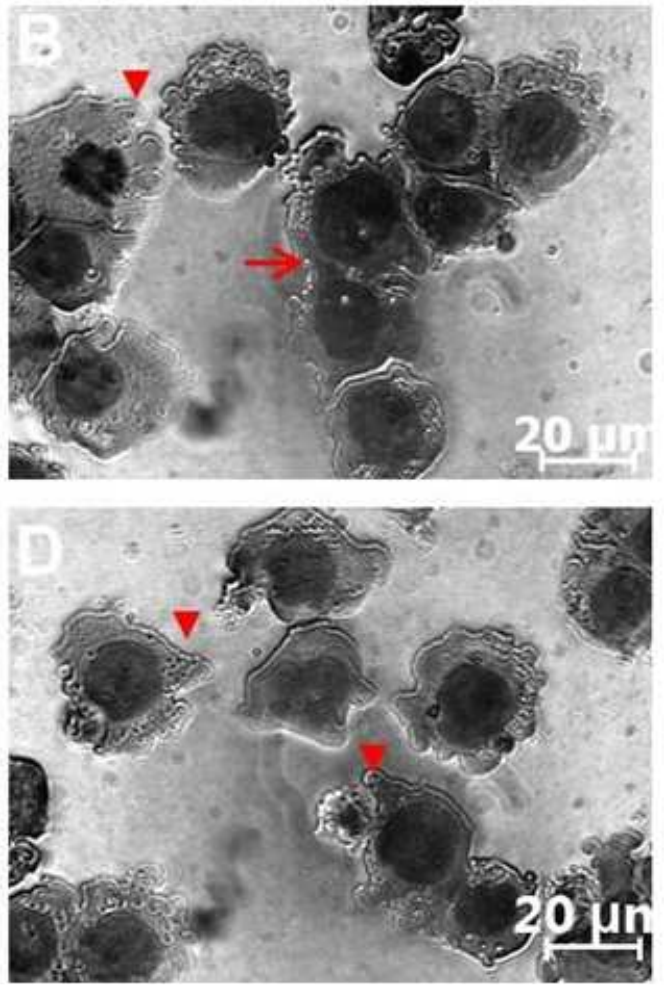

Fig. 6. DE exposure enhanced pseudopod and vacuole formation by THP-1 cells in vitro. THP-1 cells were seeded onto Millicell EZ glass slides and exposed to culture media or DE [0.1, 1 or 10\%] for $48 \mathrm{~h}$. Cells were stained with Diff Quick and viewed with a Zeiss optical microscope. Phase contrast images of THP-1 cells cultured in the presence of (A) media, (B) $0.1 \%$ DE, (C) $1 \%$ $\mathrm{DE}$ or (D) $10 \%$ DE denoting pseudopods (arrowhead) and vacuoles (arrow). Magnification $=1000 \mathrm{X}$ 


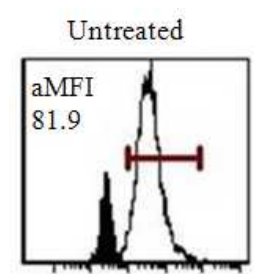

$0.1 \% \mathrm{DE}$

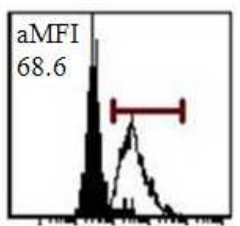

$10^{2} 10^{3} 10^{4} 10^{5} 10^{6} 10^{7}$

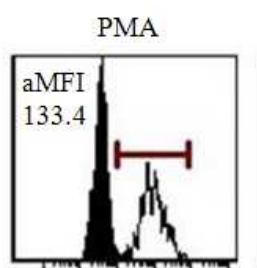

$1.0 \% \mathrm{DE}$

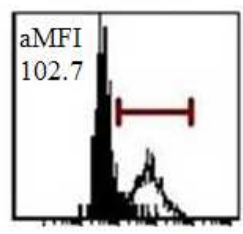

$10^{2} 10^{3} 10^{4} 10^{5} 10^{6} 10^{7}$

(A)

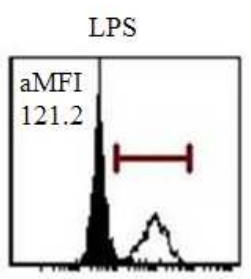

$10 \% \mathrm{DE}$

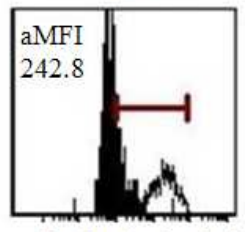

$10^{2} 10^{3} 10^{4} 10^{5} 10^{6} 10^{7}$

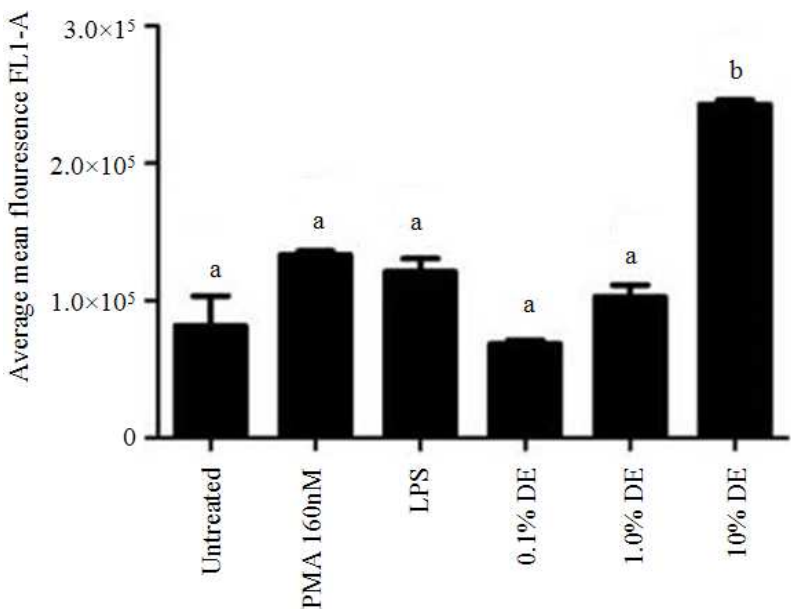

(B)

Fig. 7. Phagocytosis is enhanced in the presence of DE. THP-1 cells were left untreated or treated with PMA [160 nM], LPS [1 $\mu \mathrm{g} / \mathrm{ml}]$, or DE [0.1, 1.0 and 10\%] in the absence or presence of beads coated with FITC-labeled rabbit IgG for 24h. The degree of bead uptake was assessed by flowcytometry where (A) is a representative histogram from each treatment (filled without beads unfilled with beads). aMFI $=$ The average mean flourescence intensity of triplicate wells. Panel (B) is a graph of the aMFI; Significant differences, $\mathrm{p}<0.05$ between $10 \% \mathrm{DE}$ treated cells (b) and all other treatment conditions (a) as determined by one-way ANOVA with Bonferroni post-test. Data are represented as mean \pm SEM

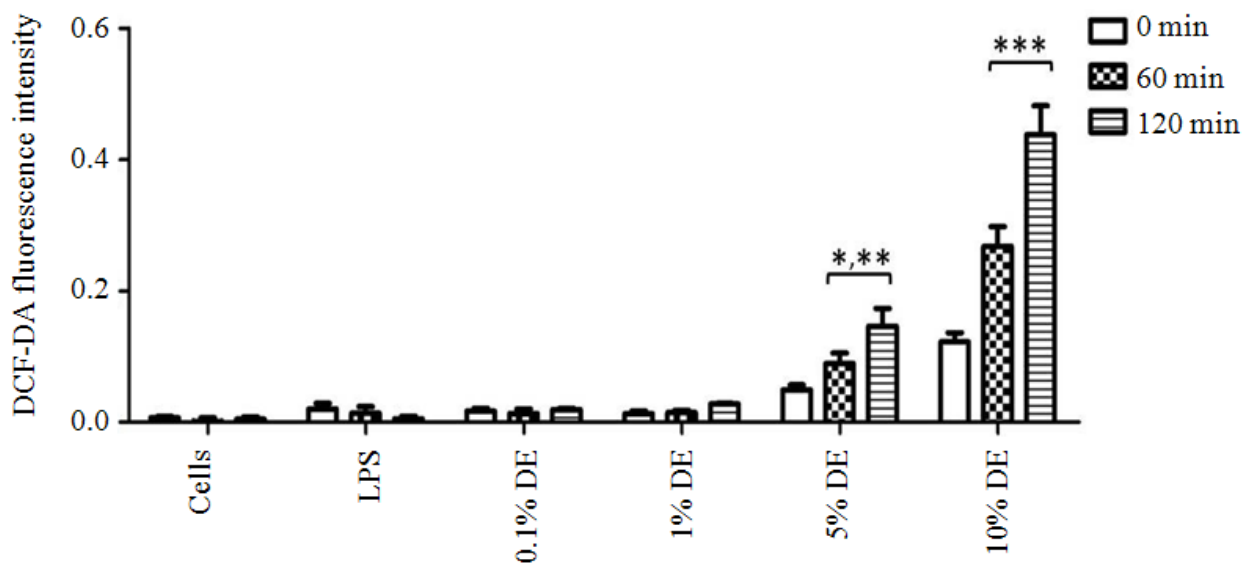

Fig. 8. DE elicits ROS production by macrophages. THP-1 cells were activated by incubation with PMA [160 nM] for $48 \mathrm{~h}$, then left untreated or incubated with $1 \mu \mathrm{g} / \mathrm{mL}$ LPS or DE [0.1, 1, 5 or $10 \%]$. After $24 \mathrm{~h}$ following stimulation with LPS or DE, cells were stained with DCF and intracellular ROS production was monitored for an additional $2 \mathrm{~h} . *, \mathrm{p}<0.05$ for $5 \%$ DE Vs. cells at $60 \mathrm{~min}$; **, p<0.001 5\% DE Vs. cells at $120 \mathrm{~min}$; ***, p<0.001 for $10 \%$ DE vs. cells at $60 \mathrm{~min}$ and 120 min. Data are shown as mean \pm SEM

This can lead to chronic lung inflammation, which can lead to diseases, such as, Hypersensitivity Pneumonitis (HP), asthma-like symptoms, dust toxicity syndrome, mucous membrane inflammation syndrome and chronic bronchitis (Charavaryamath and Singh, 2006).

Inhalation of organic dust is particularly harmful to the airway because dust absorbs gaseous substances and airborne microorganisms (Kim et al., 2008). Swine confinement facilities harbor numerous aerosol components including gases and endotoxins may adhere to dust particulates which may have a size range from $<0.01$ to $100 \mu \mathrm{m}$ with $40 \%$ of those particles being respirable; particles $\leq 5 \mu \mathrm{m}$ in aerodynamic diameter (Kirkhorn and Garry, 2000). Coarse particulate matter 
(PM; with a mean-median airway diameter, MMAD, less than $10 \mu \mathrm{m}$ ), such as present in ambient air, may exacerbate asthma and COPD. Macrophages in induced sputum of healthy volunteers that inhaled between fine PM $(2.5-10 \mu \mathrm{m})$ for $30 \mathrm{~min}$ during three separate visits, displayed significantly enhanced phagocytic activity compared to inhalation of heat inactivated fine PM (2.5$10 \mu \mathrm{m}$ ) (Alexis et al., 2006). Inhalation of $0.4 \mathrm{ppm}$ ozone significantly increased the number and expression of cell surface markers associated with innate immunity (specifically, mCD14, CD11bm CD16) and antigen presentation (CD86, HLA-DR) on airway macrophages in induced sputum in healthy volunteers compared to inhaling filtered air (Lay et al., 2007). Likewise, there was a trend for higher levels of phagocytosis by macrophages in sputum of PM2.5-10 exposed subjects compared to saline control $(\mathrm{p}=0.06)$; however, formal significance $(\mathrm{p}<0.05)$ was not observed (Alexis et al., 2006).

In the current study we report that phagocytosis of particles and ROS production by a macrophage cell line was enhanced after in vitro exposure to DE from a swine confinement facility. Furthermore, while, the current study did not provide concrete evidence of mediation of macrophage recruitment after mice were exposed to DE; however, there was evidence of phagocytic activity in vivo. We report that although total macrophage numbers did not differ from controls there were differences in the levels of macrophages with pseudopodia and vacuoles in lungs of DE exposed mice as compared to those that inhaled PBS. The formation of vacuoles occurs during phagocytosis; after the pathogen (i.e., dust) has been engulfed by macrophages. Within the cells' intestinal lamina, vacuoles or lysosomes are loaded with degradative enzymes to defend the host from any infection. In this present study, the vacuoles of macrophages present in BALF of DE-exposed mice may have assisted in the digestion of inhaled dust particles. Maturation of vacuoles into phagolysosomes for elimination of engulfed particles has been well characterized (Krajcovic et al., 2013). The current study did not provide concrete evidence of mediation of macrophage recruitment after mice were exposed to DE; however, we speculate that the dust caused macrophages within the lungs to become activated and differentiate into macrophages with pseudopods or phagocytic vacuoles. Pseudopods aid in chemotaxis, defined as the movement of pseudopods along a chemical concentration gradient either toward or away from the chemical stimulus. Elevated levels of macrophages with pseudopods may indicate enhanced directional movement in response to inhaled particulates within the DE. This is a possible indication that with the increase in macrophages with pseudopods, more DE particles were potentially captured, engulfed and phagocytosed by macrophages.

Here mice repeatedly exposed to DE displayed elevated levels macrophages and proteins in the BALF. It was found that there was a significant increase in protein concentrations between control and $12.5 \% \mathrm{DE}$ treatments. There was also a trend of higher BALF protein levels in mice that inhaled 5 and $25 \%$ DE than inhalation of PBS, although these values did not reach formal significance $(p<0.05)$. Measurement of BALF components including immune cells, microbial/host protein content, cytokines and chemokines, is the method of choice for elevating airway and lung inflammation (Elizur et al., 2008; Sagel et al., 2009). This is consistent with findings reported by Poole et al. (2007) where acute exposure to dust induces an inflammatory response demonstrated by secretion of proinflammatory cytokines; however, re-stimulation resulted in a diminished response. This observation may be explained by phenomena termed chronic inflammation adaptation response (Sundblad et al., 2009; May et al., 2012) in which repetitive dust exposure yields a less severe response in agriculture workers compared to naïve subjects. Sundblad et al. (2009) report an adaptive response in farmers (who had been exposed to a pig farm daily in the six months preceding the study) compared to controls and symptom-free cigarette smokers acutely exposed to dust in a pig barn as determined by elevated nitric oxide exhalation, nasal lavage IL-8 and sputum IL6 in smokers and control subjects compared to farmers.

Farmers may experience occupational hazards, including inhalation of endotoxins and carbon dioxide at levels above the recommended health threshold limit in SCF (McDonnell et al., 2008). After these threshold limits are exceeded, acuteand chronic airway inflammation can occur (Schierl et al., 2007). Airway inflammation can lead to loss of lung function, as measured by the decrease in forced expiratory air in one second or less (Costa et al., 2007). Poole et al. (2009b) reported increased cellular inflammation, predominated by neutrophils and neutrophils and macrophages, in mouse BALF following a single and $1 / 2$ week intranasal instillation of $\mathrm{DE}$, respectively. Mice continuously exposed to swine CAFO dust via nebulizer for $4 \mathrm{~h}$ developed BALFneutrophilia (Mueller-Anneling et al., 2006). The role of macrophages present in the lungs of mice chronically treated with DE is not known. In our model it is apparent that chronic DE exposure elicits macrophage-mediated cellular inflammation. Taken together, this information suggests that continued improvements of the workplace and provisions for health protection are necessary (McDonnell et al., 2008). Having a better understanding of the mechanisms 
involved in the mediation of chronic airway inflammation and disease to organic dust can help lead to preventative or therapeutic agents.

\section{CONCLUSION}

In conclusion, delivery of aerosolized substances to mice via nebulizer is not novel; however, to our knowledge this is the first report documenting an increase in macrophages with vacuoles and pseudopods following long-term, repeated exposure to aerosolized swine housing dust extract using an in vivo mouse model. This mouse model is distinct in that it is uncoupled from sensitization with agents such as LPS, ovalbumin or methacholine prior to the administration of swine housing dust. Furthermore, this research has shown an increase in protein in BALF, likely reflecting an increase in plasma protein exudate; however, this requires further exploration.

One limitation of the present study is that cytokines, which influence disease pathogenesis, were not determined. However, similar studies in organic dust-exposed mice or cultured macrophages have respectively shown that BALF and cell-free supernatants contains cytokines and inflammatory mediators (Poole et al., 2012). The objective of this study was to determine presence of macrophage populations in the nebulizer model of mice exposed to aerosolized SCF DE over an 8-week period in the absence of prior sensitization or tolerization. Our results show that repeated, long-term inhalation of SCF DE was successful in modulating BALF macrophage populations despite the fact that total leukocyte numbers did not vary significantly among control and DE exposed mice. The abundance of pseudopod-bearing and vacuolated macrophages in mice that inhaled DE for 8 weeks was mirrored by an acute DE exposure of THP-1 cells in vitro. Notably, significant increases in THP-1 cell phagocytosis were observed in response to incubation with DE in a dose-dependent manner. Thus, we speculate that macrophages within mouse BALF displayed enhanced phagocytosis following inhalation of DE.

Although swine farmers display low-level inflammation following acute exposures to dust, chronic lower respiratory diseases remain an issue. Studies to elucidate the role of occupational exposure to CAFO dust in onset and exacerbation of chronic non-allergic asthma, chronic bronchitis and COPD are needed.

\section{ACKNOWLEDGEMENT}

We acknowledge Zhigang $\mathrm{Xu}$ for helpful optical microscopy imaging tips. We thank Lauren L. Kloc for critical reading of the manuscript. This study was supported by the North Carolina Alliance to Create Opportunity through Education (NC Opt-Ed) program that is funded through the National Science FoundationAlliances for Graduate Education and the Professoriate (NSF-AGEP) Program and the United States Department of Agriculture-National Institute of Food and Agriculture (USDA-NIFA) Grant \# NC.X-255-5-11-120-1 (to J.T.W.) and constitutes a portion of thesis research completed as partial for a Master of Science (to R.J.P., North Carolina Agricultural and Technical State University). None of the authors have competing financial interests to disclose.

\section{REFERENCES}

Alexis, N.E., J.C. Lay, K. Zeman, W.E. Bennett and D.B. Peden et al., 2006. Biological material on inhaled coarse fraction particulate matter activates airway phagocytes in vivo in healthy volunteers. J. Allergy Clin. Immunol., 114: 1396-403. DOI: 10.1016/j.jaci.2006.02.030

Charavaryamath, C. and B. Singh, 2006. Pulmonary effectsof exposure to pig barn air. J. Occup. Med. Toxicol., 6: 10-13. DOI: 10.1186/1745-6673-1-10

Costa, M., P. Teixeira and P. Freitas, 2007. Respiratory manifestations and respiratory diseases: Prevalence and risk factors among pig farmers in Braco do Norte, Brazil. J. Bras. Pneumol., 33: 380-388. DOI: 10.1590/S1806-37132007000400006

Demanche, A., J. Bonlokke, M.J. Beaulieu, E. Assayag and Y. Cormier, 2009. Swine confinement buildings: Effects of airborne particles and settled dust on airway smooth muscles. Ann. Agric. Environ. Med., 2: 233-238. PMID: 20047256

Donham, K., J. Lee, K. Thu and J. Reynolds, 2006. Assessment of air quality at neighbor residences in the vicinity of Swine production facilities. J. Agromedicine,11:15-24. DOI: 10.1300/J096v11 n03_03

Elizur, A., C.L. Cannon, T.W. Ferkol, 2008. Airway inflammation in cystic fibrosis. Chest, 133;489-495. DOI: $10.1378 /$ chest.07-1631

Gamage, L.N.A., C. Charavaryamath, T.L. Swift and B. Singh, 2007. Lung inflammation following a single exposure to swine barn air. J. Occup. Med. Toxicol., 2:18. DOI: 10.1186/1745-6673-2-18

George, C., H. Jin, C. Wohlford-Lenane, M. ONeill and J. Phipps et al., 2001. Endotoxin responsiveness and subchronic grain dust-induced airway disease. Am. J. Physiol. Lung Cell Mol. Physiol., 2: L203-13. PMID: 11158998 
Hollingsworth, J., Z. Li, D. Brass, S. Garantziotis, S. Timberlake and A. Kim et al., 2007. CD44 regulates macrophage recruitment to the lung in lipopolysaccharide-induced airway disease. Am. J. Respir. Cell Mol. Biol., 2: 248-53. DOI: 10.1165/rcmb.2006-0363OC

Kim, K., H. Ko, H. Kim, C. Kim and Y. Kim et al., 2008. Effect of manual feeding on the level of farmer's exposure to airborne contaminants in the confinement nursery pig house. Ind. Health, 2:13843. DOI: $10.2486 /$ indhealth. 46.138

Kirkhorn, S.R. and V.F. Garry, 2000. Agricultural lung diseases. Environ. Health Perspect., 108: 705-12. PMID: 10931789

Krajcovic, M., S. Krishna, L. Akkari, J.A. Joyce and M. Overholtzera, 2013. mTOR regulates phagosome and entotic vacuole fission. Mol. Biol. Cell., 27: 3736-3745. DOI: 10.1091/mbc.E13-07-0408

Lay, J.C., N.E. Alexis, S.R. Kleeberger, R.A. Roubey and B.D. Harris et al., 2007. Ozone enhances markers of innate immunity and antigen presentation on airway monocytes in healthy individuals. J. Allergy Clin. Immunol., 120: 719-722. DOI: 10.1016/ j.jaci.2007. 05.005

May, S., D.J. Romberger and J.A. Poole, 2012. Respiratory health effects of large animal farming environments. J. Toxicol. Environ. Health B. Crit. Rev., 15: 524-41. DOI: 10.1080/10937404. 2012744288

McDonnell, P., M. Coggins, V.J. Hogan and G. Fleming, 2008. Exposure assessment of airborne contaminants in the indoor environment of Irish swine farms. Ann. Agric. Environ. Med., 2: 323-6. PMID: 19061270

Meo, S.A., S. Rasheed, M.M. Khan, S. Shujauddin and A.S. Al-Tuwaijri, 2008. Effect of cement dust exposure on phagocytic function of polymorphonuclear neutrophils in cement mill workers. Int. J. Occup. Med. Environ. Health. 21: 133-9. DOI: 10. 2478/v10001-008-0017-9

Minor, R.C., M.L. Robinson-Wright, M. Williams and S.L. Hurley, 2012. Dietary $\beta$-glucan leads to increased TNF- $\alpha$ production in the lung. Am. J. Anim. Vet. Sci., 7: 55-60. DOI: 10.3844/ajavsp.20 12.55 .60

Mueller-Anneling, L.J., M.E. O’Neill and P.S. Thorne, 2006. Biomonitoring for assessment of organic dustinduced lung inflammation. Eur. Respir. J., 27: 1096-1101. DOI: 10.1183/09031936.06.00092204

NRC, 2011. Guide for the Care and Use of Laboratory Animals. 8nd Edn., National Academies Press, Washington, DC. ISBN-13: 978-0-309-15401-7.
Poole, J.A., A.M. Gleason, C. Bauer, W.W. West and N. Alexis et al., 2012. CD11c (+)/CD11b (+) cells are critical for organic dust-elicited murine lung inflammation. Am. J. Respir. Cell Mol. Biol., 47: 652-9. DOI: $10.1165 / \mathrm{rcmb} .2012-00950 \mathrm{OC}$

Poole, J.A., G.M. Thiele, N.E. Alexis, A.M. Burrell and C. Parks et al., 2009a. Organic dust exposure alters monocyte-derived dendritic cell differentiation and maturation. Am. J. Physiol. Lung Cell Mol. Physiol., 297: L767-76. DOI: 10.1152/ ajplung.00107.2009

Poole, J.A., T.A. Wyatt, P.J. Oldenburg, M.K. Elliott and W.W. West et al., 2009b. Intranasal organic dust exposure-induced airway adaptation response marked by persistent lung inflammation and pathology in mice. Am. J. Physiol. Lung Cell Mol. Physiol., 296: L1085-L1095. DOI: 10.1152/ajplung.90622.200 .2008

Poole, J.A., T.A. Wyatt, S.G. Von Essen and J. Hervert et al., 2007. Repeat organic dust exposure-induced monocyte inflammation is associated with protein kinase C activity. J. Allergy. Clin. Immunol., 120: 366-73. DOI: 10.1016/ j.jaci. 2007.04 .033

Robinson, J.M., 2008. Reactive oxygen species in phagocytic leukocytes. Histochem. Cell. Biol. 130: 281-297. DOI: 10.1007/s00418-008-0461-4

Rodero, M.P. and K. Khosrotehrani, 2010. Skin wound healing modulation by macrophages. Int. J. Clin. Pathol., 3: 643-653. PMID: 20830235

Rylander, R., 2010. Organic dust induced pulmonary disease-the role of mould derived beta-glucan. Ann. Agric. Environ. Med., 17: 9-13. PMID: 20684476

Sagel, S.D, M.K. Sontag, F.J. Accurso, 2009. Relationship between antimicrobial proteins and airway inflammation and infection in cystic fibrosis. Pediatr. Pulmonol., 44: 402-409. DOI: 10.1002/ppul.21028

Schierl, R., A. Heise, U. Egger, F. Schneider and R. Eichelser et al., 2007. Endotoxin concentration in modern animal houses in southern bavaria. Ann. Agric. Environ. Med., 14: 129-136. PMID: 17655190

Sundblad, B.M., I. von Scheele, L. Palmberg, M. Olsson and K. Larsson, 2009. Repeated exposure to organic material alters inflammatory and physiological airway responses. Eur. Respir. J., 34: 80-88. DOI: $10.1183 / 09031936.00105308$

Underhill, D.M. and H.S. Goodridge, 2012. Information processing during phagocytosis. Nat. Rev. Immunol., 12: 492-502. DOI: 10.1038/nri3244 